of evaporation from it, and to study also the transfer of heat from fruit to air and from air to pipes. Such work is of direct value to British shipping in the transport of foodstuffs and to home producers who may wish to store their produce.

The effect of carbon dioxide in the atmosphere is not only to depress the post-climacteric respiratory activity of the fruit, but also to delay markedly the occurrence of the climacteric itself. (The climacteric is the sudden critical change in the life of the fruit when respiratory activity is doubled, and flavour and aroma are developed, the fruit attaining maturity shortly afterwards.) Apples, bananas, peaches and pears produce a substance which stimulates the onset of the climacteric in other fruit which have not yet reached this stage, but oranges and grapes do not. With the progress of senescence of apples in air, there is a steady rise in the amount of alcohol and acetaldehyde present: in an atmosphere containing little oxygen, the accumulation of these substances is slowed, so that the life of the fruit is correspondingly extended. A relationship has been found in apples between the incidence of the climacteric, the time at which the fruit is placed in cold-storage and the occurrence of low temperature breakdown. The incidence of the latter was by far the greatest in fruit transferred to cold store at the peak of the climacteric rise in respiratory activity : unfortunately, there is no practical means as yet whereby growers can detect when their fruit enters the elimacteric.

These are a few of the directions, indicated by Sir Frank Smith in his lecture at Aberdeen, in which our knowledge of the properties of foodstuffs is advancing. Such knowledge can only be of inestimable benefit to mankind, and will be for ever associated with the name of the late Sir William Hardy.

\title{
Mathematical Aspects of the Propagation of Light*
}

By Prof. H. M. Macdonald, o.B.E., F.r.s.

$\mathrm{F}^{\mathrm{A}}$ ARADAY, like Fresnel, appears to have thought of light in terms of geometrical relations, while Maxwell sought to construct a mechanical model the motions of which will resemble those which constitute light.

Starting from Faraday's ideas, the problem of the propagation of a magnetic disturbance in free space can be approached in a direct manner. There are three vectors involved-the electric current at a point in the space, the magnetic force at the point, and the electric force at the point. The relation between the electric current and the magnetic force is given by Ampère's law, and the relation between the magnetic force and the electric force is given by Faraday's law. It should be noted that Ampere's law was established initially for steady electric currents ; its extension to the case where the electric currents are varying is a result of Faraday's work. Assuming, with Faraday, that the phenomena of light and of electricity have a common origin, Fresnel's law of transversality, that the vectors which specify the disturbance are perpendicular to the direction of propagation, will hold for the propagation of an electric or a magnetic disturbance as well as for light.

These three laws are sufficient to determine the circumstances of the propagation of a magnetic disturbance in free space. It follows that for plane waves the direction of the vector $j$, the time rate of increase of which is the electric current, at a point coincides with the direction of the electric

"From the presidential address entitled "Theories of Light" to Section A (Mathematical and Physical Sciences) of the British Association, delivered at Aberdeen on September 7 . force $E$ at the point, and the relation between $E$ and $j$ is $E=4 \pi V^{2} j$, where $V$ is the velocity of propagation of a magnetic disturbance in free space. Further, if the changes which constitute the disturbance satisfy the laws of dynamics, the potential energy per unit of volume is $\frac{1}{2} E j$-that is, $E^{2} / 8 \pi V^{2}$ in electromagnetic units-and, if $E_{1}$ is the same electric force in electrostatic units, the potential energy is $E_{1}^{2} / 8 \pi$; therefore $E=V E_{1}$, that is, the velocity of propagation is the velocity by which an electric force expressed in electrostatic units must be multiplied to convert it into electromagnetic units; or since the product of an electric charge and the electric force on it, being a mechanical force, is the same in both systems of units, the velocity of propagation is the velocity by which an electric charge expressed in electromagnetic units must be multiplied to convert it into electrostatic units.

The Lagrangian function of the changes which belong to the propagation of an electric or magnetic disturbance in free space is the difference of a kinetic energy function and a potential energy function. The potential energy function is the function given above-the kinetic energy function depends on the electromagnetic momentum and the electric current at a point; the contribution from an element in the neighbourhood of a point cannot be expressed in terms of one vector: it depends on the electric currents throughout space. On this theory, the rate of transfer of energy from a source emitting waves of one frequency is steady, and not oscillatory as on an elastic solid theory.

Consistently with the foregoing, the effect of 
material media, so far as electric and magnetic phenomena are concerned, can be represented by a distribution of electric currents and of magnetic currents throughout the space occupied by the material media. These electric current and mag. netic current distributions can be supposed to be due to electric charges and to magnetic particles which are in motion, and it follows from the electrodynamical equations, when these current distributions are taken account of, that the current distributions can be represented by a distribution of electric and magnetic oscillators throughout the space occupied by the material media.

Further, the magnetic field due to a distribution of electric and magnetic currents inside a closed surface at any point outside this closed surface can be expressed in terms of the components of the electric and magnetic forces tangential to the surface-that is, any distribution of electric and magnetic currents inside a closed surface produces the same magnetic field at points outside the surface as a distribution of electric and magnetic currents on the surface which is determined by the components of the magnetic and electric forces tangential to the surface at points on it, but a knowledge of the magnetic field external to a closed surface does not determine the distrib. ution of electric and magnetic currents inside the surface which is producing the magnetic field.

When the states of motion belonging to the electric and magnetic current distributions in the material medium are steady states of motion, the material medium is in a state of relative equilibrium ; but, when an electric or magnetic disturbance is being propagated in the material medium, these steady states of motion will be disturbed and, under certain conditions, the effect of the disturbance will be to set up small oscillations about the steady states of motion. A material can be regarded as being perfectly transparent for a disturbance the only effect of which is to set up small oscillations about the steady states of motion. A condition for this is that none of the frequencies involved in the disturbance is equal to or nearly equal to any of the natural frequencies belonging to the steady states of motion.

Fresnel's relations between the amplitudes of the incident, the transmitted, and the reflected waves when a train of waves is incident on the surface separating two transparent media follow on this hypothesis, and also Fresnel's results for the propagation of waves in crystalline media. It should be noticed that on this hypothesis the electric and magnetic forces at a point in a material medium which appear in the equations are not, the total electric and magnetic forces at the point, but the parts of them which are due to the disturbance.

Faraday's results for the rotation of the plane of polarisation by an imposed magnetic field when light is being propagated in a non-magnetic transparent medium follow immediately from the above hypothesis without making any additional assumptions.

Further, on the same hypothesis there will be ranges of frequencies for which a material medium is transparent, the extent of such a range will depend on the intensity of the disturbances, and between any two consecutive ranges there will be a range of frequencies for which the medium is not transparent, and the mathematical treatment of the effect of disturbances involving these frequencies will require additional hypotheses.

The theory advanced above is not a mechanical theory of light in the sense that it is possible to construct a machine the motions of which will resemble the motions involved in the propagation of light. The form of the electrokinetic energy function raises the question whether all the time rates of change involved in the propagation of a magnetic disturbance can be represented by moving points, and whether every time rate of change associated with physical phenomena involves change of position in space. It may be necessary to contemplate time rates of change which do not involve change of position in space although they satisfy the laws of dynamics. In this connexion it is of interest to observe that a result of Faraday's laws is that, when there are electric currents in a system of circuits which are in motion, the kinetic energy function does not contain terms which involve the product of an electric current and a velocity, a result which Maxwell verified experimentally.

A possible hypothesis is that physical phenomena are due to the interaction of time rates of change which satisfy the laws of dynamics, and the Lagrangian function in that case would be a homogeneous quadratic function of all the time rates of change. In actual cases only some of the changes are being observed, and the Lagrangian function which is obtained from the experimental evidence is a modified Lagrangian function where the unobserved changes are supposed to be eliminated. In certain cases, this function will be expressed as the difference of a kinetic energy and a potential energy function; an important case is that where the unobserved changes appear in the original Lagrangian function as velocities only and there are no product terms which involve a velocity belonging to the observed and a velocity belonging to the unobserved changes. There are also cases where the modified function is of this form approximately. 